\title{
TREATMENT-RESISTANT DEPRESSION: ALL-CAUSE AND CAUSE-SPECIFIC EXCESS MORTALITY
}

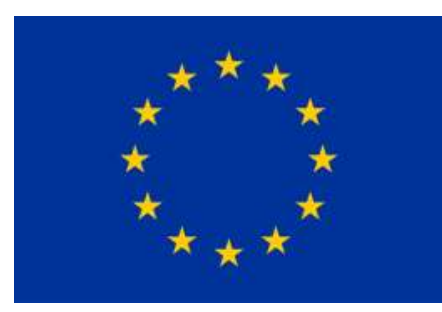

\author{
Kathrine Bang Madsen 1,2,3, Oleguer Plana-Ripoll1,2, Liselotte Vogdrup Petersen 1,2, \\ Katherine L. Musliner ${ }^{1,2}$, Jean-Christophe Philippe Debost ${ }^{1,2}$, Trine Munk-Olsen 1,2,3 \\ 'National Centre for Register-based Research, Department of Economics and Business Economics, \\ Aarhus BSS, Aarhus University, Aarhus, Denmark \\ 2iPSYCH, The Lundbeck Foundation Initiative Psychiatric Research, Aarhus, Denmark \\ ${ }^{3} \mathrm{EU}$ project 754740 - MOODSTRATIFICATION
}

INTRODUCTION Depression is associated with excess mortality, but it is not yet known how treatment-resistance influences life expectancy. Approximately one-third of patients with depression are estimated to be treatment-resistant (TRD) and consequently can expect poorer prognostic outcomes.
Our aim was to examine the differences in all-cause and causespecific mortality and life expectancy, estimated by mortality rate ratios (MRR) and Life Years Lost (LYL) in individuals with TRD compared to non-TRD individuals first identified in primary care in a nationwide population study.
METHODS All individuals born in Denmark with an antidepressant prescription at age 18-69 years between Jan 1, 1996 and Dec 30, 2012 were identified in the Danish National Prescription Registry $(\mathrm{N}=$ 562,648). TRD was defined a priori based on established definitions; at least to shifts in medication classes, including augmentation or combination. Information on date and primary cause of death was obtained from the Danish Registry of Causes of Death. MRRs were estimated with Cox regression adjusted for age at first prescription, calendar year and comorbidity and LYL by the Life Years Lost method.

RESULTS Of the population, $27,920(5 \%)$ individuals were identified with TRD. The study cohort was observed over 5,863,165 person-years (TRD: 278,002 ), in which there were 71,374 deaths (TRD: 2,901). Adjusted MRRs between individuals with and without TRD was highest for suicide and accidents in both men (aMRR: $3.13,95 \%$ Cl 2.67; 3.66 and aMRR: $1.30,95 \% \mathrm{Cl} 1.03$; 1.65 ) and women (aMRR: 6.06 , $95 \% \mathrm{Cl} 5.08 ; 7.23$ and aMRR: $1.67,95 \% \mathrm{Cl} 1.26 ; 1.65)$.
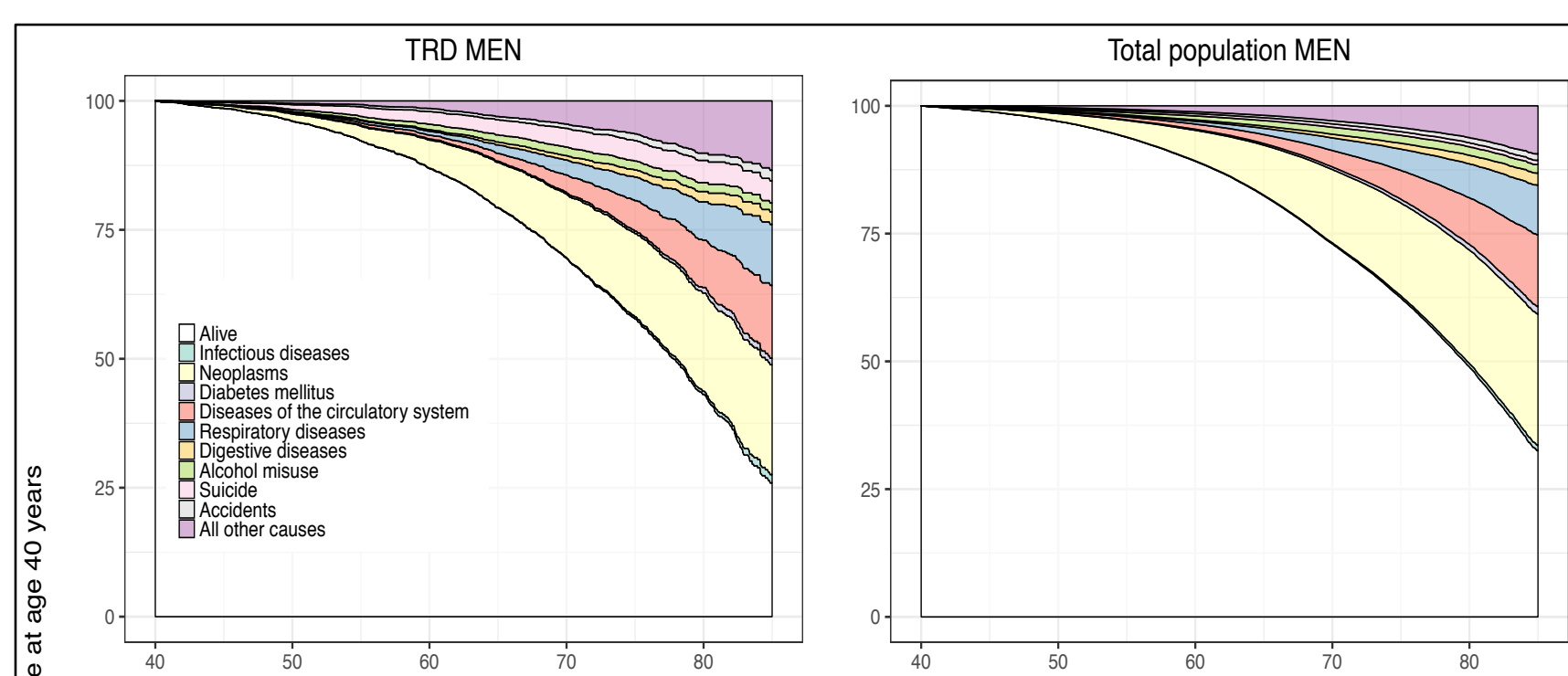

TRD WOMEN

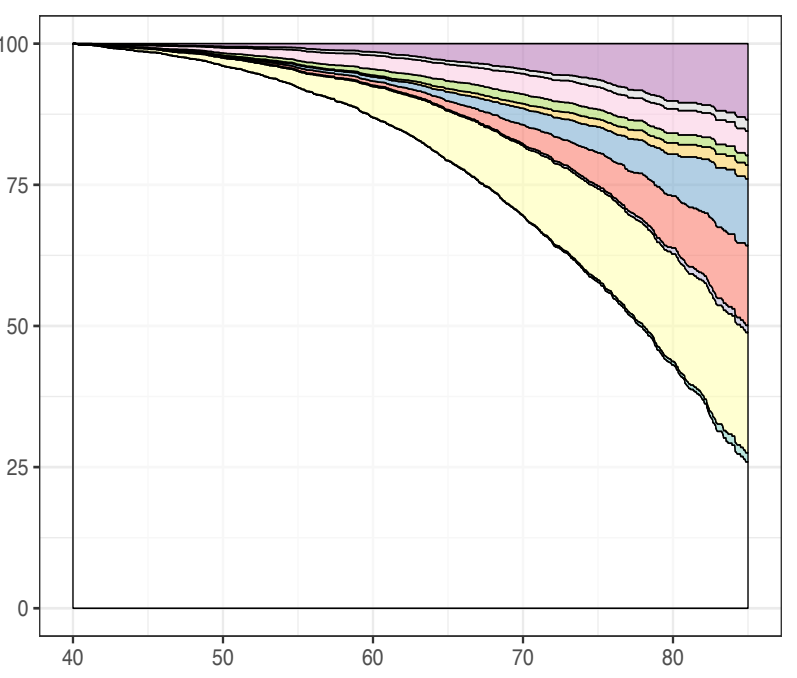

Total population WOMEN

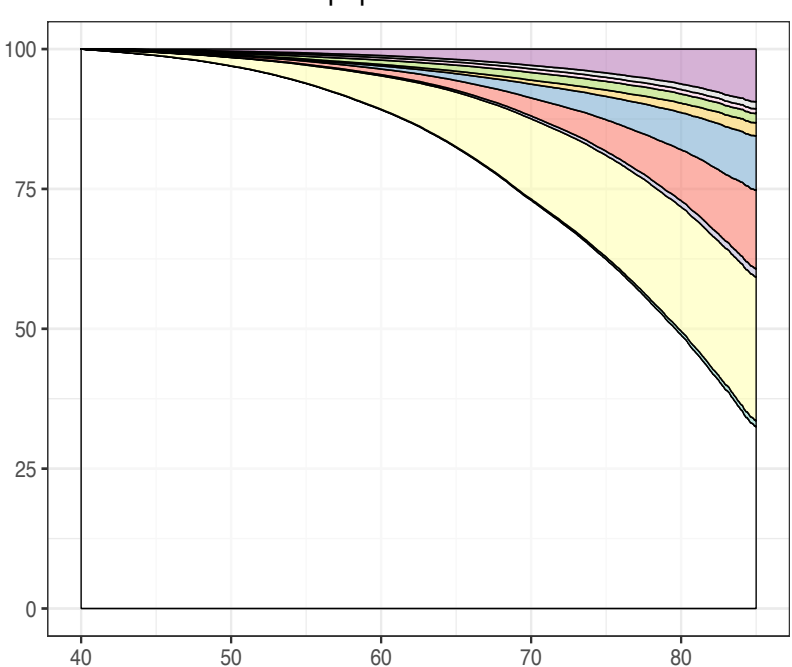

Age in years

Table 1. Life years lost before age 90 and excess life years lost by cause of death in men and women with TRD

\begin{tabular}{|c|c|c|c|c|c|c|}
\hline & \multicolumn{3}{|l|}{ MEN } & \multicolumn{3}{|c|}{ WOMEN } \\
\hline & \multicolumn{3}{|c|}{ Life-years lost before age 90} & \multicolumn{3}{|c|}{ Life-years lost before age 90} \\
\hline & TRD & $\begin{array}{l}\text { Total } \\
\text { cohort }\end{array}$ & $\begin{array}{l}\text { Excess life } \\
\text { years lost }\end{array}$ & TRD & $\begin{array}{l}\text { Total } \\
\text { cohort }\end{array}$ & $\begin{array}{l}\text { Excess life } \\
\text { years lost }\end{array}$ \\
\hline All causes & 18.17 & 12.26 & 5.91 & 13.92 & 12.56 & 1.36 \\
\hline Infectious diseases & 0.21 & 0.16 & 0.05 & 0.19 & 0.16 & 0.03 \\
\hline Neoplasms & 5.21 & 5.22 & -0.01 & 4.57 & 5.37 & -0.8 \\
\hline Diabetes mellitus & 0.54 & 0.26 & 0.28 & 0.21 & 0.27 & -0.06 \\
\hline Diseases of the circulatory system & 4.21 & 2.22 & 1.99 & 2.21 & 2.23 & -0.02 \\
\hline Respiratory diseases & 1.29 & 1.52 & -0.23 & 1.78 & 1.52 & 0.26 \\
\hline Digestive diseases & 0.37 & 0.4 & -0.03 & 0.44 & 0.41 & 0.03 \\
\hline Alcohol misuse & 1.11 & 0.41 & 0.7 & 0.49 & 0.43 & 0.06 \\
\hline Suicide & 1.94 & 0.24 & 1.7 & 1.28 & 0.27 & 1.01 \\
\hline Accidents & 0.77 & 0.27 & 0.5 & 0.45 & 0.3 & 0.15 \\
\hline All other causes & 2.52 & 1.56 & 0.96 & 2.3 & 1.6 & 0.7 \\
\hline
\end{tabular}

Figure 1. Probabilities of survival and deaths from different causes for men and women with TRD and the total population alive at age 40 years and followed until 85 years.

THE LIFE YEARS LOST METHOD Differences in life expectancy were estimated with the Life Years Lost method.

For the TRD group the LYL measure quantifies the average remaining life expectancy from the age at TRD restricted to a reference age of 90 years. For the total population, the LYL measure quantifies the average remaining life expectancy subtracted from 90 years for subjects alive at ages corresponding to the age at TRD for those with TRD.

The difference in Life Years Lost between the two populations denote excess Life Years Lost and is interpreted as the number of years that individuals with TRD lose in excess of that found in the total population

(Plana-Ripoll et al. Estimating excess Life Years Lost among patients with a given disease or condition: a tutorial. In press).
CONCLUSION To our knowledge, this is the first study to investigate the excess mortality of individuals with TRD first identified in primary care and quantify which causes of death contributed to the excess life-years lost between individuals with and without TRD. Individuals with TRD have a significant excess mortality compared to individuals who respond to treatment. For men, diseases of the circulatory system, suicide, alcohol misuse and accidents accounted for the major shares of life-years lost in individuals with TRD, while for women, it was suicide, respiratory diseases and accidents.

\section{WHAT THIS STUDY ADDS}

- The first study to investigate excess mortality in individuals with TRD first identified in primary care.

- Individuals with TRD have a significant excess mortality compared to individuals who respond to treatment, mainly due to suicide.

- Men and women with TRD lose on average almost 6 and more than 1 excess life years, respectively, from all-cause mortality. 